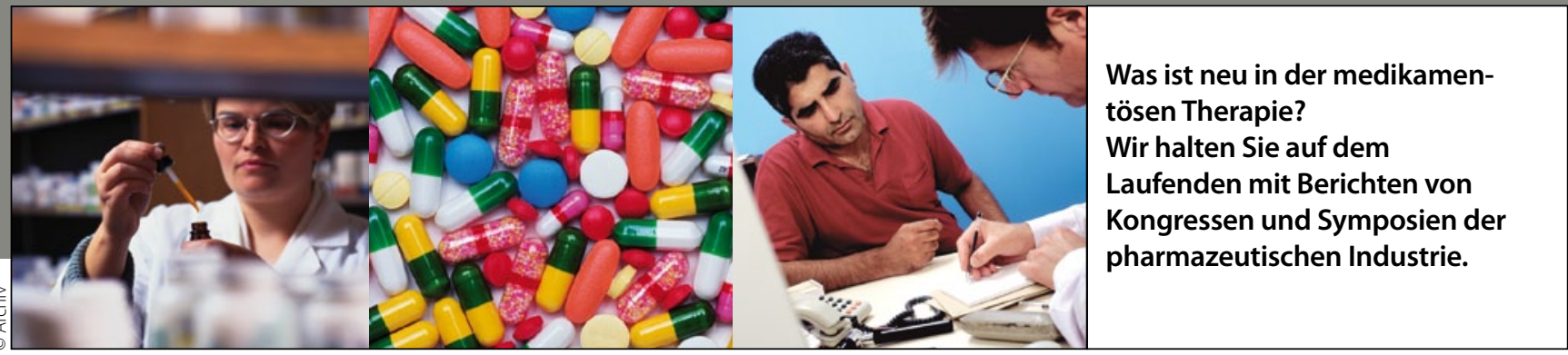

Koronare Herzkrankheit

\title{
Mikrovaskuläre Störungen werden häufig unterschätzt
}

Bei KHK-Patienten, die keine Koronarstenosen aufweisen oder nach Koronarintervention symptomatisch bleiben, kann eine Therapie mit Ranolazin lohnen, das einer Kalziumüberladung der Zelle entgegenwirkt und damit die diastolische Herzfunktion verbessert.

— Kardiale Ischämien entstehen aufgrund eines Missverhältnisses zwischen myokardialem $\mathrm{O}_{2}$-Bedarf und $\mathrm{O}_{2}$-Angebot. Ursache sind nicht immer makrovaskuläre Koronarstenosen, erklärte Dr. Dirk Westermann, Berlin. Dies veranschaulichen zwei Zahlen: In einer Studie war bei knapp 400000 Patienten mit Angina pectoris untersucht worden, wie oft eine obstruktive KHK vorliegt im Sinne einer 50\%-Hauptstamm- oder 70\%-Stenose in einem anderen Koronargefäß. Ergebnis: bei nur $41 \%$. Eine andere Studie belegt, dass über $40 \%$ aller KHK-Patienten nach Bypass-OP oder PTCA weiter pektanginöse Beschwerden aufweisen.

Neben der makrovaskulären obstruktiven KHK gibt es noch mikrovaskuläre Ursachen der Angina pectoris, die prognostisch bedeutsam sind und therapeutisch weder mit einer Revaskularisierung noch mit klassischen Antianginosa behandelt werden können, so Westermann. Dazu zählen:

- endotheliale Dysfunktion

- Small vessel disease

- diastolische Dysfunktion

- lonen-Dysfunktion.

Gerade die lonen-Dysfunktion auf zellulärer Ebene gewinnt im Kontext von koronaren Ischämien, Herzinsuffizienz und Rhythmusstörungen zunehmend an Be- deutung, wie Dr. Samuel Sossalla, Göttingen, ausführte. Im Fokus steht der Natriumeinstrom in die Zelle in der Spätphase des Aktionspotenzials, der sog. INA-late.

\section{Später Natriumeinstrom stört diastolische Funktion}

Wenn unter einer Ischämie oder im Rahmen einer Herzinsuffizienz in pathologischer Weise zu viel Natrium in die Zelle eingeschleust wird, kommt es konsekutiv zu einer intrazellulären Kalziumüberladung, erklärte Sossalla. Diese ist schlecht für die Myokardfunktion: Aktin- und Myosinfilamente bleiben auch in der Diastole kontrahiert, was zu einer Relaxationsstörung, einem Anstieg der Wandspannung und zu mikrosvaskulären Durchblutungsstörungen führen kann. Das Resultat: weiter erhöhter $\mathrm{O}_{2}$-Bedarf bei verschlechterter $\mathrm{O}_{2}$-Versorgung - ein Circulus vitiosus. So- mit erklärt sich, wie der späte Natriumeinstrom Ischämie verursacht.

Ranolazin (Ranexa ${ }^{\oplus}$ ) hemmt spezifisch den späten Natriumeinstrom, aber nicht den Spitzen-Natriumeinstrom. Die Substanz weist einen antianginösen Wirkmechanismus auf, der sich von allen anderen KHK-Therapeutika grundlegend unterscheidet. Ranolazin ist in der Lage, bei Patienten mit limitierender KHK die Zeit bis zur Angina und Ischämie deutlich hinauszuzögern, ohne dabei Herzfrequenz oder Blutdruck zu beeinflussen. Die Substanz ist indiziert bei Patienten mit KHK, die entweder keine relevanten Koronarstenosen oder aber nach Revaskularisierung weiterhin pektanginöse Beschwerden aufweisen.

\section{- Dr. med Dirk Einecke}

Quellen: Symposium „Kardiale Ischämien in der Praxis - Mehr als KHK?", Düsseldorf, Oktober 2011 (Veranstalter: Berlin Chemie)

\section{Knifflige Differenzialdiagnose besonders zu Beginn KHK, Cholezystitis oder Herpes zoster?}

\begin{abstract}
- Die Prodromalphase des Herpes zoster ist charakterisiert durch unspezifische Symptome: Krankheitsgefühl mit Abgeschlagenheit, Müdigkeit, beginnende Schmerzen, Dysästhesien, intermittierende oder permanente brennende Missempfindungen, z.T. heftige Berührungsschmerzen. In dieser Phase ist die Diagnose häufig schwierig, da die unspezifischen Schmerzen je nach Dermatombefall z. B. auch auf ein akutes Koronarsyndrom, eine Perikarditis, Cholezystitis, akute abdominale Erkran-
\end{abstract}

kung, Koliken der Leber/Niere oder auf eine Nervenerkrankung, Schmerzen im Augenbereich auf ein Glaukom hinweisen können.

Leichter zu diagnostizieren scheint der Herpes zoster, wenn die typischen Hauterscheinungen - gruppiert stehende Bläschen - sichtbar sind. Bläschen im Mundoder Genitalbereich können jedoch z.B. auch auf Insektenstiche zurückzuführen sein oder auf Impetigo, Candidose bzw. Dermatitis herpetiformis hinweisen. Außerdem sollte Folgendes bedacht werden: 